\title{
Mechanical and morphological properties of injection-molded rice husk polypropylene composites
}

\begin{abstract}
In this work, the investigation of the physical, mechanical, and morphological properties of the rice husk flour/polypropylene composites was performed utilizing various filler loadings and coupling agent. Five levels of filler loading (35, 40, 45, 50, and $55 \mathrm{wt} \%)$ were designed. In addition, to help the interaction between fiber and polypropylene matrix, struktol coupling agent was added to the composites. All of tensile strength, Young's modulus, flexural strength, flexural modulus, and impact strength properties of the composites were carried out. Moreover, the $50 \mathrm{wt} \%$ filler-loaded composites had optimum tensile strength, flexural strength, and flexural modulus, whereas the $35 \mathrm{wt} \%$ of filler loading case was the best regarding Young's modulus, flexural strength, flexural modulus, and impact strength. Furthermore, the scanning electron microscope results demonstrate that as filler loading increases, more voids and fiber pullout occur.
\end{abstract}

Keyword: Injection molding; Mechanical properties; Natural fibers; Polypropylene; Rice husk 